\title{
Predicting asphaltene aggregate structure from molecular dynamics simulation: comparison to neutron total scattering data
}

\author{
TF Headen and MP Hoepfner
}

\section{Published version information}

Citation: TF Headen and MP Hoepfner. "Predicting asphaltene aggregate structure from molecular dynamics simulation: comparison to neutron total scattering data." Energy \& Fuels, vol. 33, no. 5 (2019): 3787-3795.

DOI: 10.1021/acs.energyfuels.8b03196

This document is the unedited author's version of a Submitted Work that was subsequently accepted for publication in Energy \& Fuels, copyright (02019 American Chemical Society, after peer review. To access the final edited and published work see DOI above.

Please cite only the published version using the reference above. This is the citation assigned by the publisher at the time of issuing the AAM. Please check the publisher's website for any updates. 


\title{
Predicting asphaltene aggregate structure from
}

\section{molecular dynamics simulation: Comparison to}

\author{
neutron total scattering data.
}

Thomas F. Headen*1, Michael P. Hoepfner²

${ }^{1}$ ISIS Neutron and Muon Source, Rutherford Appleton Laboratory, Harwell Campus,

Didcot, United Kingdom, OX11 0QX

2 Department of Chemical Engineering, The University of Utah, 50 South Central

Campus Drive, Salt Lake City, Utah 84112, United States

KEYWORDS: Asphaltene, Molecular Dynamics, Neutron Total Scattering, Small-Angle

Scattering

ABSTRACT: Molecular dynamics simulation is quickly growing in popularity as a technique for understanding asphaltene aggregate structure and dynamics. However, 
verification of the results of simulations against experimental data has, to date, been sparse. Here we present total scattering data from Athabasca asphaltenes, both as a solid and dispersed at high concentrations in deuterated 1-methylnaphthalene. The advantage of total scattering is that the expected scattering can be calculated from knowledge of the atomic positions in the system of question, meaning that simulation and experiment can be directly compared. We find that the molecular dynamics simulations for model mono-disperse systems reproduce the general form of the scattering curves well, particularly for the slope and shape for the small-angle scattering curve of dispersed asphaltenes. However, we find a number of limitations in the MD techniques as commonly used in the literature, specifically the size of the aggregates formed is considerably smaller than observed from the scattering data. We identify two main causes of this discrepancy, namely the limited box size that can be reasonably simulated and the lack of molecular polydispersity.

Introduction 
The simulation of model asphaltene molecules by molecular dynamics (MD)

simulation is a burgeoning field in asphaltene science, allowing aggregation processes and structures to be studied in unprecedented detail. ${ }^{1-13}$ These include studies of aggregation in good and poor solvents ${ }^{1}$, at oil-water interfaces ${ }^{2-6}$, on solid surfaces ${ }^{7,8}$, during $\mathrm{CO}_{2}$ flooding ${ }^{6,9}$ and studies correlating molecular structure to aggregation ${ }^{10,11}$ and the effectiveness of aggregation inhibitors. ${ }^{12,13}$ There is huge potential in such studies as a way to understand the molecular level structure and kinetics behind asphaltene precipitation and fouling. Ultimately the goal of atomistic simulations studies of asphaltenes is to be able to predict asphaltene phase behavior. If it is possible to build a "digital" version of a crude oil based on chemical analysis, one could explore at what conditions the asphaltenes are unstable, whether two crudes could be incompatible or screen a series of different aggregation inhibitors structures in silico. However, the accuracy of the results of these simulations relies on both the molecular models used correctly representing asphaltene molecular structures, and the forcefields accurately portraying the non-covalent interactions present. 
To have sufficient confidence in the results obtained, benchmarking against experimental data is vital. Force-fields are commonly parameterized through optimization against experimental condensed phase properties (heat of vaporization, density) and/or results of ab initio calculations of representative small molecules. ${ }^{14-16}$ For example, in most cases a general aromatic carbon will be derived from benzene and other smaller aromatics. For the simulation of asphaltenes, the assumption is that the parameters for condensed polyaromatic carbons will be well represented by a single "aromatic-type" carbon. It should be recognized that many simulation force-fields are developed for the study of biomolecules and/or relatively simple liquid molecules. The extension of these force-fields (e.g., OPLS ${ }^{17}$ ) to larger aromatic Polyaromatic Hydrocarbons (PAHs) remains relatively untested, save for some preliminary studies. ${ }^{18}$ Neutron and X-ray scattering can provide a strong experimental benchmark for solution structure as it can be directly calculated from molecular simulation trajectories from Fourier inversion of the weighted sum of the radial distribution functions in the system ${ }^{19}$. Additionally, molecular simulation is also a valuable tool in analysis of liquid 
structure from scattering data ${ }^{20,21}$. Analysis of the complex scattering patterns generated by neutron/X-ray scattering of real systems can be greatly assisted by the additional constraints that must be fitted in the system when considering a molecularlevel perspective (e.g. bonded interactions and interaction energies). One example of the application of simulation techniques to understand experimental scattering results of molecular liquids is the Empirical Potential Structure Refinement (EPSR) method. ${ }^{22}$ In short, this method starts with a standard atomistic Monte-Carlo simulation of the system in question using standard force-fields. The initially predicted scattering pattern from the molecular-level simulation is then calculated and compared to experimental data. To improve confidence in structural predications and to reduce the uncertainty associated with underspecified systems, the simulated experimental scattering of different isotopologues (e.g., $\mathrm{H}_{2} \mathrm{O}$ and $\mathrm{D}_{2} \mathrm{O}$ ) are compared. The difference between the simulated and experimental scattering patterns are then used to calculate an additional empirical atom-atom potential which is included in the simulation. This approach is applied iteratively until a close match between simulated and experimental scattering is obtained, and several thousand experimentally consistent ensemble molecular positions 
are used to investigate all aspects of the structure in a liquid system. Results from

EPSR can then be used to benchmark force-fields in the ability to predict liquid

structure. ${ }^{21,23}$ Our recent studies of liquid naphthalene ${ }^{18}$ have indicated that standard

"aromatic carbon" potentials may not be sufficient to correctly predict liquid structure of

polyaromatic hydrocarbons. These EPSR studies of liquid PAHs are a "bottom-up"

approach, testing the underlying methods using simple systems. In this manuscript, we

present the initial findings of an alternative "top-down" methodology. Direct application

of the EPSR approach is currently unfeasible for asphaltenes, as tens of thousands of

unique empirical formulae have been detected with mass spectrometry techniques ${ }^{24}$,

with an unknown number of unique isomers. Alternatively, the expected scattering from

MD simulations of asphaltene systems under the same conditions (temperature,

pressure, and concentration) can be calculated and compared to those studied

experimentally by neutron scattering. This approach provides direct insight into how well

common MD methods used to date ${ }^{1,9}$ can predict the structure of solid asphaltenes and

concentrated asphaltene solutions as observed through wide $Q$-range neutron total

scattering. The level of confidence in simulation predictions is limited as the primary 
means of validating previous simulations is to qualitative comparisons with the Yen-

Mullins mode ${ }^{25}$, which does not specify the geometry of asphaltene self-associations.

The direct comparison of simulated and experimental structures provides novel insight into the extent to which existing simulation strategies successfully predict asphaltene structures and provides pathways for additional study and improvement. Herein we present a preliminary simulation study of three types of asphaltene molecular structures as used in our previous works ${ }^{26,27}$. The structures used have been chosen to exemplify the range of molecular architecture and functionalities present within asphaltenes. This approach is a simplification, ignoring the vast molecular polydispersity of asphaltenes ${ }^{24,28}$. With each petroleum source containing an effectively infinite number of compounds, how many molecules are required to be included in a simulation to provide sufficient polydispersity in molecular properties to differentiate between the characteristics of two crude oils or asphaltene samples? To date this question has not been answered and current attempts to include significant polydispersity in asphaltene molecular types and composition indicate a that a long $(\sim \mu \mathrm{s})$ simulation with a large number of asphaltene molecules ( $>300$, but only 9 unique) are necessary to 
appropriately represent the self-assembled structure of asphaltenes. ${ }^{29}$ As such, computational power with fully-atomistic simulations may be insufficient currently to include a sufficient number of asphaltenes as to obtain a representative sample of the real system. An alternative approach, that we have adopted here, is to differentiate between some molecular archetypes and understand how these broad classes of asphaltene molecules behave (e.g., island vs. archipelago). In this investigation we provide the first-ever comparison between the simulated structure of asphaltene types and their measured self-assembled structure on the molecular length scale using neutron scattering measurements. Finally we take a first step towards a more realistic simulation by using a mix of all three molecular structures.

\section{Experimental Methods}

Wide Q-range neutron total scattering was conducted at the Near and InterMediate Range Order Diffractometer (NIMROD) ${ }^{30}$ at the ISIS spallation neutron source. The instrument exploits the time-of-flight of the neutrons from the pulsed source to utilize 
neutrons over a wide wavelength range, $0.05-12 \AA$. Forward scattering detectors covering $40^{\circ}-0.5^{\circ}$ are optimized for collection of total scattering data from hydrogenous samples as they minimize inelasticity effects. The overall simultaneous momentum transfer $(Q)$ range available is $0.02-50 \AA^{-1}$, spanning the traditional techniques of smallangle neutron scattering (SANS) and neutron diffraction. This allows both atomistic and mesoscale structure to be measured simultaneously. Asphaltene aggregation has been extensively studied by both SAXS and SANS, ${ }^{31-33}$ and while a variety of models are able to fit the data, ${ }^{33,34}$ the only universal results are that they form aggregates in good solvents of radii up to $\sim 100 \AA$ and that these aggregates are diffuse as shown by having a mass fractal dimension in the range 1.6-3 and entraining a significant amount of solvent in the aggregate. However atomistic level intramolecular detail of asphaltene aggregation can only be inferred from small-angle scattering data, not measured directly, due to an insufficiently high maximum $Q$.

For wide $Q$-range neutron total scattering the measured intensity is reduced to the scattering differential scattering cross section, $F(Q)$, which is a weighted sum of all the partial (atom-atom) structure factors in the system, $S_{\alpha \beta}(Q)$. 


$$
F_{i}(Q)=\sum_{\alpha, \beta \geq \alpha}\left(2-\delta_{\alpha \beta}\right) c_{\alpha} c_{\beta} b_{\alpha} b_{\beta}\left(S_{\alpha \beta}(Q)-1\right),
$$

where $c_{\alpha}$ is the atomic fraction of species $\alpha, b_{\alpha}$ is the neutron scattering length of atom $\alpha$, and $Q=4 \pi(\sin \theta) / \lambda$ (i.e. the magnitude of the momentum change vector of the scattered neutrons). The partial structure factor can be directly calculated from the pair distribution function of the atom pair in question, $\mathrm{g}_{\alpha \beta}(\eta)$ by a Fourier transform:

$$
S_{\alpha \beta}(Q)-1=\frac{4 \pi \rho_{0}}{Q} \int_{0}^{\infty} r\left[g_{\alpha \beta}(r)-1\right] \sin (Q r) d r
$$

Therefore, if one knows the positions of all the atoms in the system, the total scattering, $F(Q)$, can be calculated. Note that it is not possible to go the other way, and calculate all the pair distribution functions unambiguously from the data - unless there are as many datasets as there are partials in the system (which is only possible for simple systems such as water or benzene). In the present study, we can use molecular simulations of model asphaltene molecules, from which pair distribution functions, partial structure factors and total differential scattering cross sections can be calculated and compared to the data. A separate paper will report comparative structural analysis 


\begin{abstract}
directly from the neutron scattering data from a wider set of asphaltene samples and experimental conditions (e.g., temperature).
\end{abstract}

Asphaltenes were precipitated from Athabasca bitumen by the addition of heptane at a ratio of $40: 1$ by weight. The mixture was homogenized by stirring for 24 hours, and the asphaltenes were separated by centrifugation at $3472 \mathrm{~g}$ for 10 minutes. Solid asphaltenes were washed for 24 hours in a Soxhlet extractor with pure heptane then dried in vacuum oven at $80{ }^{\circ} \mathrm{C}$ for 4 days. As Athabasca bitumen contains inorganic solids, an additional step was taken to isolate the asphaltenes. The solid asphaltene samples were diluted to a $4.8 \mathrm{wt}$ \% solution in toluene and centrifuged at $5000 \mathrm{~g}$ for 270 minutes to remove the inorganic solids. The toluene was then removed by forced convection and then subsequent drying in a vacuum oven at $80{ }^{\circ} \mathrm{C}$ to obtain the solid asphaltene sample used for experimentation.

Two types of sample are used in the present study: Solid precipitated asphaltenes and a $25 \mathrm{wt} \%$ solution of asphaltenes in deuterated 1-methylnaphthalene-d10 (Sigma, 98 atom $\%$ D). Samples were loaded into flat plate TiZr null scattering alloy cells with a 1 $\mathrm{mm}$ and $2 \mathrm{~mm}$ path lengths used for solution and solid samples respectively. The 
temperature of the cell was controlled in the range $20-180^{\circ} \mathrm{C}$ using a circulating oil-bath.

Neutron scattering from the samples was measured using a $30 \times 30 \mathrm{~mm}$ beam size for a minimum of 2 hours. The measured neutron scattering was reduced to the interference differential scattering cross section, $F(Q)$ using the GudrunN programme. ${ }^{35}$ This programme merges the time-of-flight scattering from all detectors to a single $Q$ scale, normalizes to a $3 \mathrm{~mm} \mathrm{VNb}$ plate calibration standard, subtracts scattering from the sample container and empty instrument and applies corrections for beam attenuation and multiple scattering. For samples containing light hydrogen, which is particularly the case for solid asphaltenes, attention must be paid to careful subtraction of inelasticity effects, which is achieved through application of an iterative correction developed by Soper. ${ }^{36,37}$ Figure 1 shows data from NIMROD for a $25 \mathrm{wt} \%$ solution of Athabasca asphaltenes in 1-methylnaphthalene-d10. The $\mathrm{x}$-axis $\mathrm{Q}$ value can be thought of as an inverse distance metric. At high $Q$ values ( $>3 \AA^{-1}$ ) the observed interference scattering is dominated by short distance correlations i.e. intra-molecular structure, that does not change with temperature. Intermediate $Q$ values $\left(1 \AA^{-1}<Q<3 \AA^{-1}\right)$ are dominated by local inter-molecular correlations and low $Q$ values $\left(<1 \AA^{-1}\right)$ reveal any clustering or 
aggregation behavior of molecules. There is a clear reduction in clustering with increasing temperature (lower levels of small angle scattering) and increased spacing and disorder in the intermolecular interactions (increased breadth and shift to lower $Q$ for a peak at $\left.\sim 1.5 \AA^{-1}\right)$.

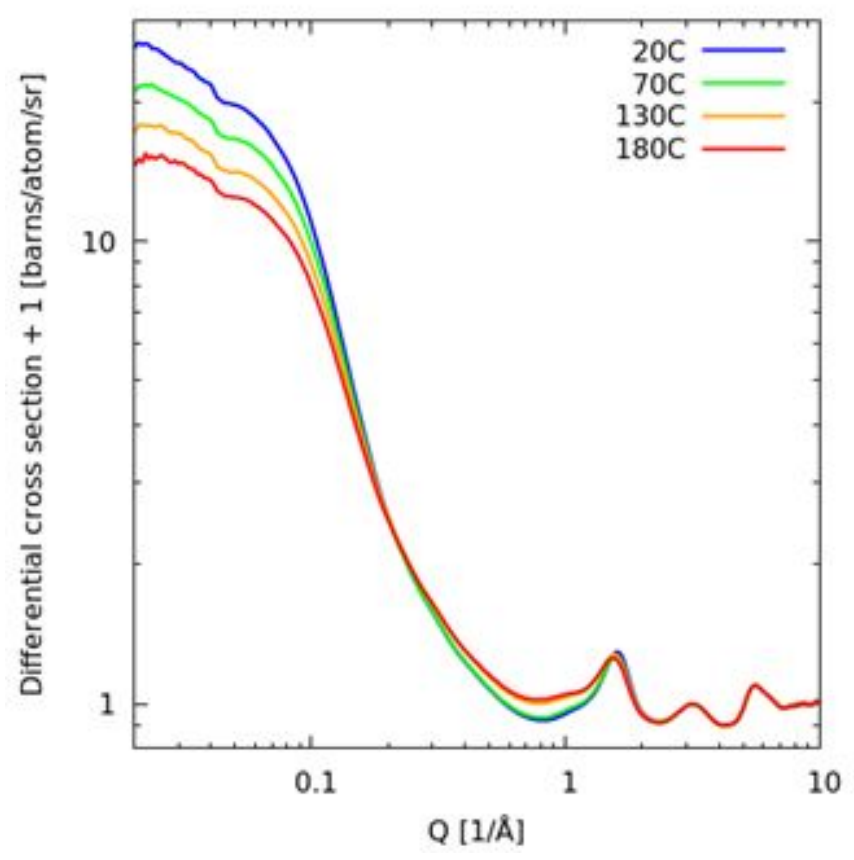

Figure 1: Neutron total scattering data for a $25 \mathrm{wt} \%$ solution of Athabasca asphaltenes in d-1-methylnaphthalene

\section{Simulation Methods}


Following previous works ${ }^{1,27}$ we use well-defined classical intra- and intermolecular potentials of the OPLS-AA force-field family ${ }^{17,38}$, these include both the atomistic Lennard-Jones parameters and the partial charges used. The OPLS force field has been shown to work well for benzene in reproducing liquid structure data ${ }^{23}$. It is important to note that improper-dihedrals must be used with aromatic structures in order to keep the aromatic ring reasonably flat. Simulation cells were constructed by random placement and rotation of asphaltene molecules followed by random placement and rotation of sufficient solvent molecules (if required) to give the required concentration. This procedure was carried out at low density to reduce the probability of molecular overlap and/or allow for pre-assembly of asphaltene aggregates. A short initial energy minimization step was used to remove any high energy structures formed, after which the system is allowed to reach equilibrium density by running an MD simulation in the isobaric-isothermal NPT ensemble. For the simulation of solid asphaltenes, simulation boxes were prepared at $<5 \%$ of their standard density. A longer (5ns) initial NVT simulation was run to allow asphaltene molecules to aggregate, and the lower density allowed aggregates to form. After which the system was "shrunk" to the equilibrium 
density using the NPT ensemble. In some instances, a higher pressure was used to force the simulation box to shrink in a reasonable time, with the systems always being re-equilibrated at 1 bar prior to initial NVT accumulation runs at $400 \mathrm{~ns}$ for solution systems and $80 \mathrm{~ns}$ for solid systems. Analysis of the initial simulations carried out at $27^{\circ} \mathrm{C}$ showed that these were trapped in a local energy minimum with little movement of the molecules in reasonable simulation timescales, therefore a simulated annealing approach was used to allow more of the thermodynamic ensemble to be covered. For the solution phase simulations in 1-methylnapthalene, simulations at $180^{\circ} \mathrm{C}$ showed molecular motion and gave smooth scattering patterns at low-Q indicating sufficient sampling of the ensemble. Therefore for the room temperature simulations at least 4 different simulation snapshots, spaced over the course of the $400 \mathrm{~ns}$ simulation (e.g. at $100,200,300$ and $400 \mathrm{~ns}$ ) were used as a new starting point. These simulation boxes were cooled to $27^{\circ} \mathrm{C}$ over $10 \mathrm{~ns}$ by linear adjustment of the simulation reference temperature, followed by $5 \mathrm{~ns}$ of equilibration at $27^{\circ} \mathrm{C}$. Each simulation box was then separately allowed to reach equilibrium density over a 2ns NPT simulation, followed by a 20ns NVT accumulation simulation from which scattering curves were calculated and 
averaged over all simulation boxes. For simulations of solid asphaltenes, the output atomic coordinates of the initial $80 \mathrm{~ns}$ simulation was taken and simulated at $1000 \mathrm{~K}$ at constant volume (NVT) for a minimum of 40ns, this allowed significant molecular movement. A minimum of four snapshots were taken over the last half of the simulation (e.g. at $25,30,35$ and $40 \mathrm{~ns}$ ). Each simulation box is then allowed to reach equilibrium density (2ns NPT) followed by a 20ns NVT accumulation run. Temperature and pressure were maintained using the Nose-Hoover ${ }^{39,40}$ and Parinello-Rahman ${ }^{41,42}$ algorithms, respectively. A pressure of 1 bar was used throughout this study unless otherwise stated.

The GROMACS MD simulation code was used for all simulations ${ }^{43}$ using the leap-frog MD algorithm and the Verlet pair list scheme for neighbour searching. A time step of $1 \mathrm{fs}$ was used for all simulations and bond lengths were kept rigid using the LINCS algorithm ${ }^{44}$. Cubic periodic boundary conditions were employed to approximate infinite bulk behaviour. The cut-off of the non-bonded interactions was set at $1 \mathrm{~nm}$, with a standard dispersion correction for energy and pressure employed to account for longer 
range dispersion interactions. Long-range electrostatic interactions were dealt with using the Particle Mesh Ewald procedure ${ }^{45}$.

Three different types of model asphaltene molecule were used as part of this study: a small and a large continental asphaltene and an archipelago asphaltene (see Figure 2).

These structures have been previously studied extensively by atomistic simulation ${ }^{1,11,27}$, and have quite different structures allowing comparison of the intermolecular structures formed. Continuing the naming used in ref 1 we label these asphaltenes $C, D$ and $E$ respectively. These structures have a very similar atomic composition to that obtained from the elemental analysis of the asphaltene sample used in this study, as shown in Table 1. 


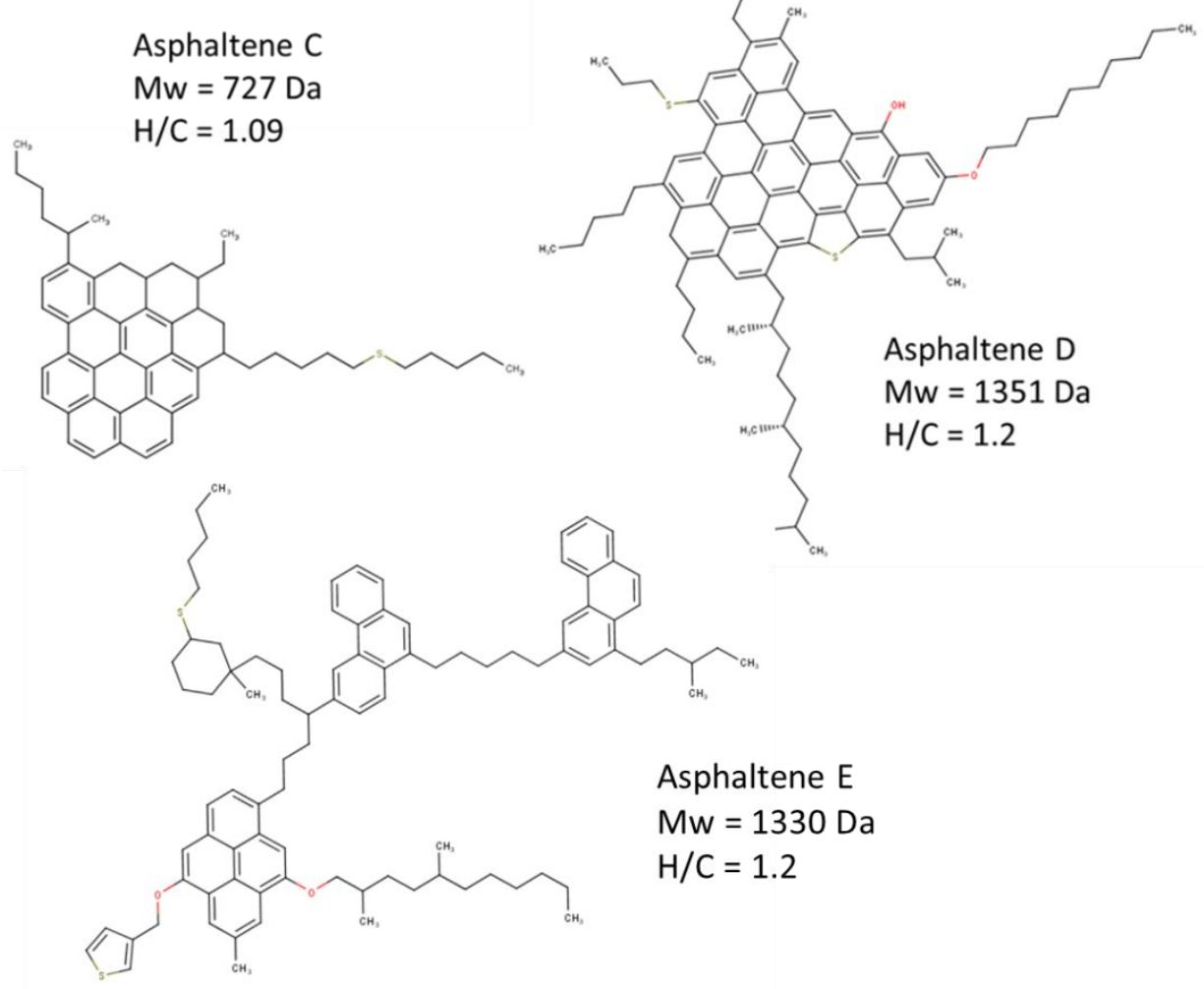

Figure 2: Structures of the asphaltene models used in this study

Table 1: Elemental composition of asphaltene sample compared to elemental composition of the three asphaltene structures used in this study:

\begin{tabular}{l|llll} 
ELEMENT & $\begin{array}{l}\text { EXPERIMENTAL } \\
\text { ATOM\% }\end{array}$ & ASPHL C & ASPHL D & ASPHL E \\
\hline C & $45 \%$ & $47 \%$ & $45 \%$ & $44 \%$ \\
H & $52 \%$ & $52 \%$ & $53 \%$ & $54 \%$ \\
N & $1 \%$ & $0 \%$ & $0 \%$ & $0 \%$ \\
S & $2 \%$ & $1 \%$ & $1 \%$ & $1 \%$
\end{tabular}




\begin{tabular}{l|lccc}
$\mathrm{O}$ & $1 \%$ & $0 \%$ & $1 \%$ & $1 \%$ \\
$\mathrm{H} / \mathrm{C}$ & 1.16 & 1.12 & 1.19 & 1.25
\end{tabular}

The solid asphaltene simulations used asphaltene C, D and E structures $(400,300$ and 300 molecules respectively), and a mixed simulation contain approximately the same mass of each of these three asphaltenes $(193,104,104$ molecules). For the dissolved asphaltenes three systems were simulated: 200 molecules of asphaltene C with 2866 molecules of 1-methylnaphthalene, 110 molecules of asphaltene E with 2866 molecules of 1-methylnaphthalene, and a mixed system containing 67,33 and 33 of asphaltenes C, D and E respectively with 2866 molecules of 1-methylnaphthalene. All solution systems were simulated at $27^{\circ} \mathrm{C}$ and $180^{\circ} \mathrm{C}$ so that temperature effects could be investigated. The neutron scattering was calculated from the saved simulation trajectories using the "sq" function in the dlputils analysis code ${ }^{46}$.

\section{Results \& Discussion}




\section{Solid Precipitated Asphaltenes}

In Figure 3 we show a portion of the total scattering data for solid precipitated

Athabasca asphaltenes compared to the calculated scattering for the four MD

simulations of solid asphaltenes using the small continental model (asphaltene $\mathrm{C}$ ), the

large continental model (asphaltene D), the archipelago asphaltene (asphaltene E), and

the mixed system. 


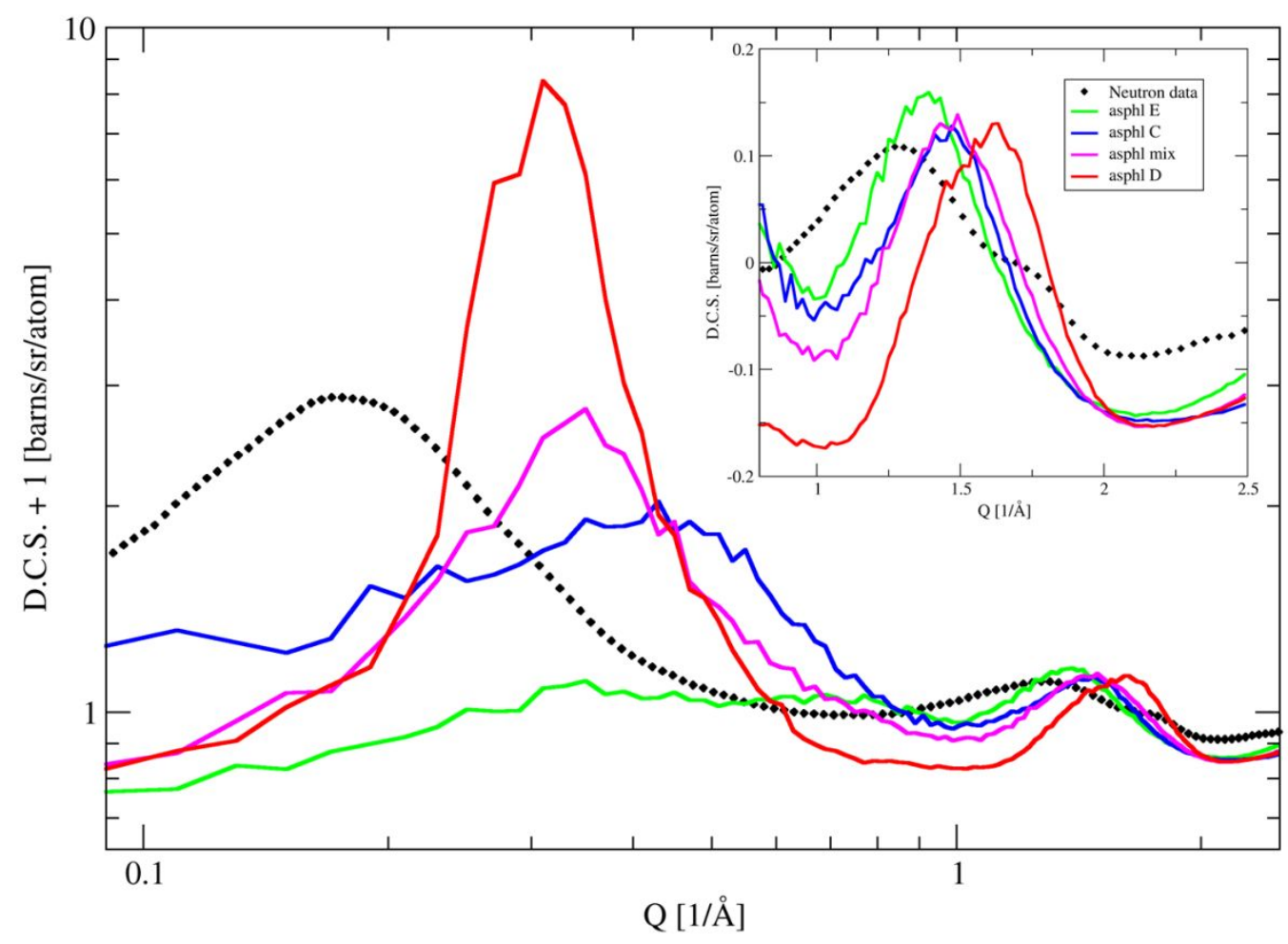

Figure 3: Neutron total scattering data from solid precipitated Athabasca asphaltenes (black diamonds) compared to MD simulations from asphaltene molecular models: a large continental asphaltene ( $\mathrm{D}$ - red line) and a small continental asphaltene ( $\mathrm{C}$ - blue line), an archipelago asphaltene ( $\mathrm{E}$ - green line) and a mixture of all three (magenta line) 
In the low $Q$ portion of the neutron data there is a clear peak in the data at $Q=0.18 \AA^{-}$

${ }^{1}$ corresponding to a non-crystalline local correlation with spacing $d \sim 35 \AA$. The most

likely structural feature for this correlation is spacing between regions of condensed aromatic cores, as also reported previously by Sirota. ${ }^{47}$ The contrast for this peak in SANS experiments arises from differences in scattering length densities between hydrogen-rich aliphatic chains and hydrogen-poor aromatic cores. Scattering contrast could also come from pores form in the structure, however we do not see pores in any of the solid asphaltene simulations. All simulations containing continental asphaltenes replicate this small angle peak feature but at much higher $Q$, indicating that the separation between aromatic and aliphatic rich regions in the simulation is too small compared to the real system. In other words, the aromatic regions of separate asphaltene nanoaggregates are packed too closely together in the simulation compared to the experimental measurements. Comparing the simulations using the continental models, the larger continental asphaltene $\mathrm{D}$ has the lowest $Q$ peak, giving the best match to the data in terms of aromatic region correlation distance. However, the height of the peak is greater for asphaltene $D$, indicating a greater level of order than is 
perhaps present in the real system. Snapshots of the simulations showing only the aromatic carbons of the asphaltene molecules (Figure 4) clearly show a greater degree of aromatic clustering for asphaltene $\mathrm{D}$, as might be expected due to the larger aromatic core size. 

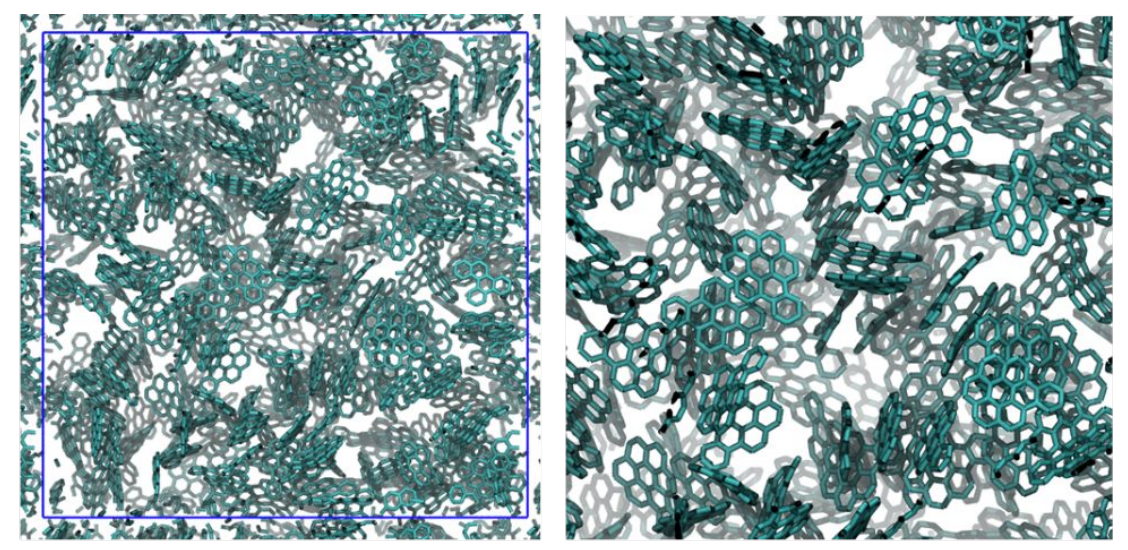

Small continental asphaltene $\mathrm{C}$

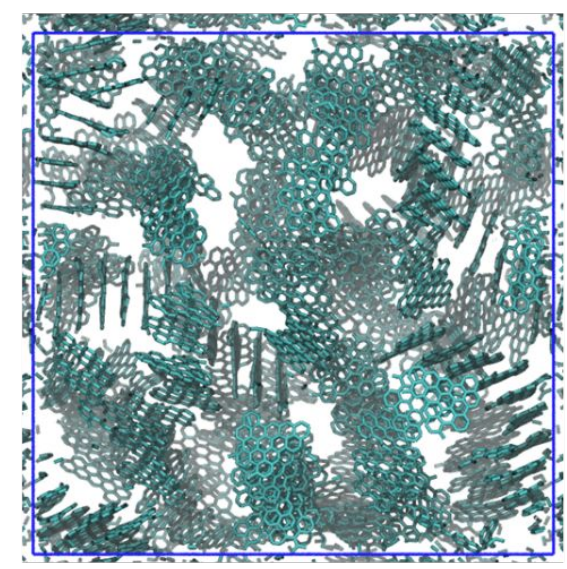

Large continental asphaltene D

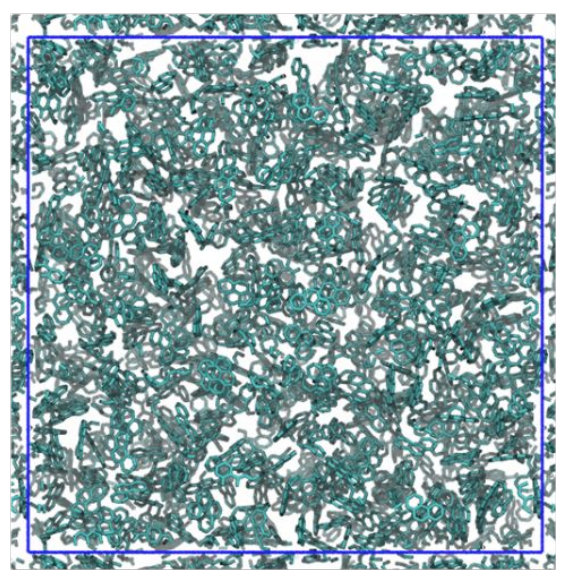

Archipelago asphaltene $\mathrm{E}$

Figure 4: Snapshots of simulations of solid asphaltenes using a small continental model asphaltene ( $\mathrm{C}$ - top) and a large continental model asphaltene ( $\mathrm{D}$ - bottom left) and an archipelago asphaltene (E - bottom right), only the aromatic carbons are displayed showing difference in scale of aromatic-aliphatic separation. 
The extent of clustering was investigated by calculating cluster size histograms, following the same approach used in a previous MD study of asphaltene solutions ${ }^{1}$ using the clustsize utility provided in the GROMACS suite of programs ${ }^{43}$. The size of all the clusters in the system is calculated for each frame by using a minimum cut-off distance, a group of molecules are considered to be in a cluster if there is a continuous path of contacts which are below this cut-off. In this case only aromatic carbons were considered and a cut-off $0.4 \mathrm{~nm}$ was used, corresponding closely to the minimum on the Lennard-Jones potential for aromatic carbon. While this approach does not explicitly confirm the aromatic cores are in a stacked arrangement, it prevents the inclusion of perpendicular arrangements, as the presence of peripheral atoms $(\mathrm{H}$, alkyl chains) will force the $\mathrm{C}-\mathrm{C}$ distance beyond this cut-off. In Figure 5 we plot the histogram as a probability of finding an asphaltene molecule in a cluster of size $N$, averaged over simulations. For the smaller asphaltene $\mathrm{C}$ model, a majority of the simulation occurs with nearly all the aromatic cores in contact as a continuous network "cluster". Close inspection of the trajectory snapshots reveals how this is possible through highly displaced and/or slightly tilted "stacking" between the aromatic cores (Figure 4 top 
right). For the larger asphaltene $D$ no such large-scale clustering is observed, with the stacks being localized with much greater overlap. This distribution is broad with a similar probability of finding an asphaltene molecule in a cluster of up to 60 molecules in size, indicating relatively large, regular stacks, but no continuous cluster.

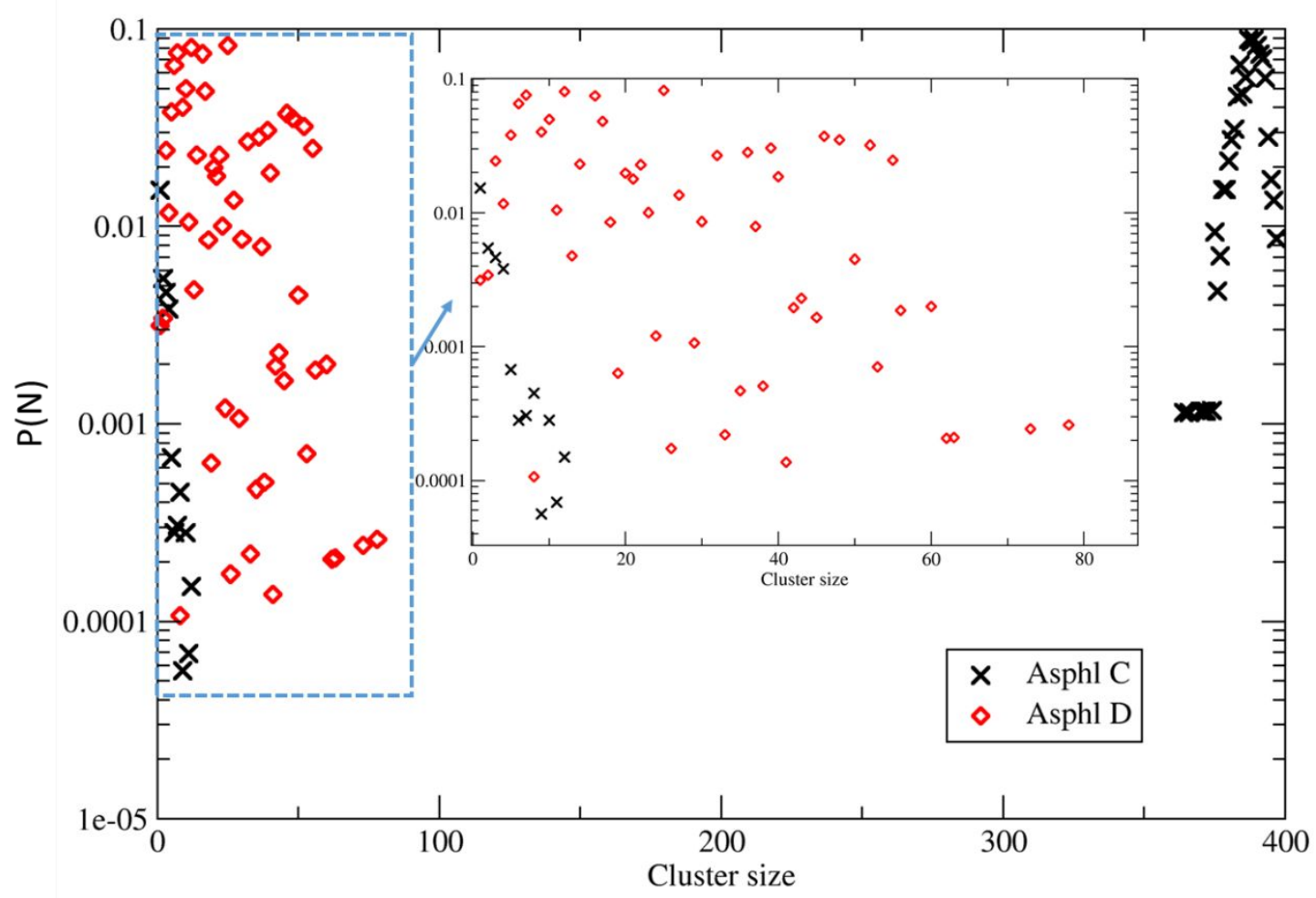

Figure 5: Cluster size histogram for aromatic cores from simulations of solid asphaltenes using a large and small asphaltene models ( $D$ and $C$ respectively). 
The intermolecular correlations are most prevalent in the intermediate Q-region (Figure 3 insert). Here the experimental neutron scattering data show two broad overlapping peaks at $Q \sim 1.2 \AA^{-1}$ and $1.7 \AA^{-1}$. Similar diffraction data have long been observed for precipitated asphaltenes, ${ }^{48,49}$ with the interpretation of the data being that the lower $Q$ peak, labeled the $y$-peak, is due to correlations between aliphatic chains and/or other non-specific nearest neighbor interactions, and the higher $Q$ peak, labeled the [002]-peak being due to the stacking of aromatic cores, in similarity to the interlayer structure of graphite. All simulations show only one peak in the intermediate $Q$ range. The simulated scattering for larger asphaltene $D$ model does show much greater scattering towards the higher $Q$ [002] peak, with higher levels of parallel stacking of aromatic cores are clear from the simulation snapshots given in Figure 4, however there is no clear $y$ peak as seen in the experimental scattering. Conversely, the scattering from the archipelago asphaltene $E$ model comes closest to matching the $\mathrm{Y}$-peak, with the small island model asphaltene $\mathrm{C}$ somewhere in-between the two. Interestingly the simulation of the mixed system also gives one peak, close to that for asphaltene $\mathrm{C}$ We 
could consider that a good match would come from a suitably weighted some of the simulated scattering from asphaltene $\mathrm{D}$ and $\mathrm{E}$. This suggests that in the experimental system there is greater phase separation between aromatic and aliphatic rich molecules, such that on a local level like is surrounded by like.

In order to allow quantitative comparisons of the level of aromatic stacking in the simulations, the radial distribution function for aromatic carbons only was calculated. These results are presented in Figure 6 and exclude contributions from atoms on the same molecule (i.e., intermolecular features only). The radial distribution functions demonstrate a clear short-range correlation for aromatic carbons in asphaltene $D(r=$ $4.25 \AA$ ) that is almost completely absent for the smaller asphaltene $\mathrm{C}$ and non-existent for the archipelago asphaltene $E$ 


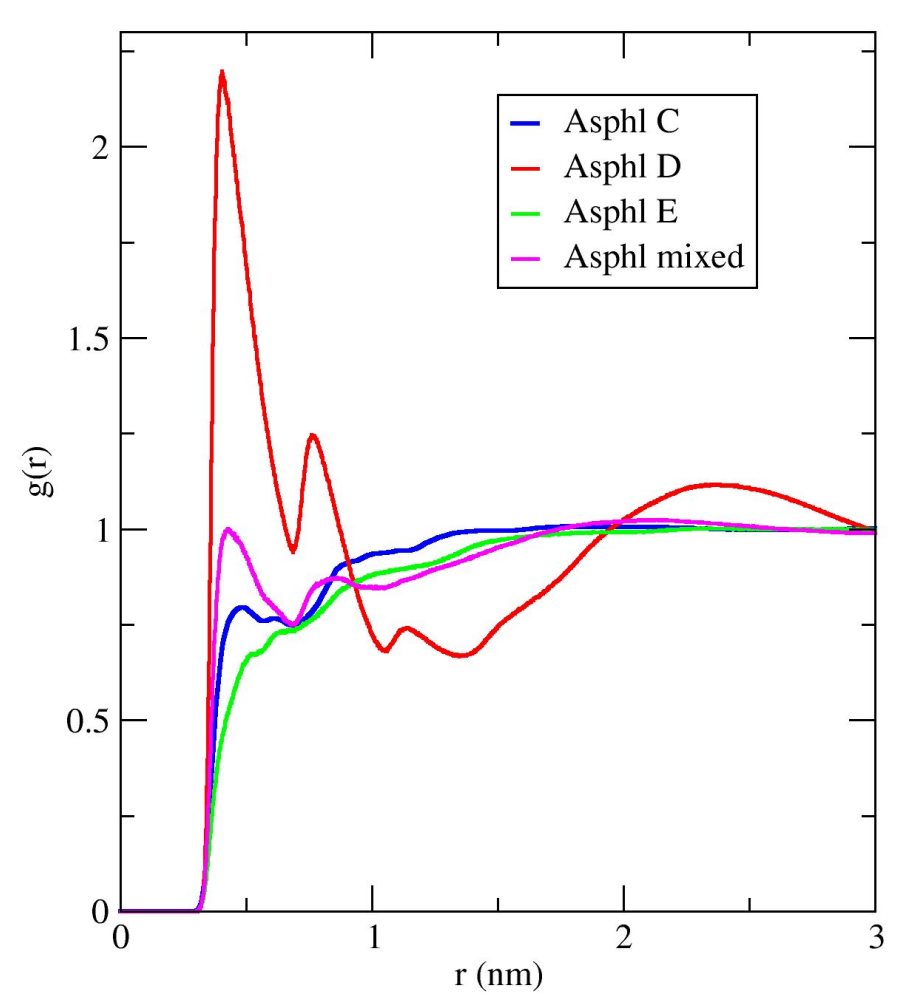

Figure 6: Radial distribution function, $g(r)$, between aromatic carbons on different asphaltene molecules for MD simulations of solid asphaltenes.

It is clear that the simulations of solid asphaltene using three different continental asphaltenes and a simple mixed system do not replicate the experimental total scattering patterns well. They do show a low- $Q$ correlation peak, but at too high a $Q$ (too closely packed aromatic cores). This observation, at least partially, may be due to the limited size of the MD simulation box used. Another likely shortcoming is the lack of 
structural polydispersity in the models used. The variation in molecular structure of asphaltenes is incredibly large ${ }^{50}$ with each molecular structure probably being unique within a certain sample. We suggest the inclusion of vast molecular polydispersity will allow larger length-scale separation into aromatic-rich and aliphatic-rich regions. However this will require very large simulation boxes combined with very long simulation times, which may be prohibitively expensive for atomistic MD. This wide polydispersity, and subsequent nano-phase separation into aliphatic and aromatic-rich regions can also help explain the lack of separation of the intermediate $Q$ peak in the simulation into the two broad features ( $Y$ and [002]) seen in our data and elsewhere. ${ }^{48}$ The nano-scale separation allows increased aliphatic-aliphatic and aromatic-aromatic contacts for nearest neighbor molecules therefore showing more distinct $\mathrm{Y}$ and [002] peaks than is observed in our structurally monodisperse simulations.

\section{Asphaltene Solutions in 1-methylnaphthalene}

A comparison of neutron scattering data to simulated scattering for asphaltenes C, E and mixed solutions in 1 -methylnaphthalene at $27^{\circ} \mathrm{C}$ and $180^{\circ} \mathrm{C}$ are shown in Figure 7 , 
and snapshots of these simulations at $27^{\circ} \mathrm{C}$ are shown in Figure 8. Looking at the low $\mathrm{Q}$ region first, it is clear that the general form and shape of the small angle scattering curve is correct, however the simulated structures are significantly smaller than the experimental system revealed by the Guinier plateau occurring at a much higher $Q$ value compared to the data. The good match between the shape and slope of the curve (before the plateau, $Q>0.19 \AA^{-1}$ ) indicates that the simulation correctly predicts the fractal nature of the asphaltene aggregation - the slope of the SANS curve being directly related to the dimensionality of the scattering object ${ }^{51}$. One should note that at such high concentrations there will be significant cluster overlap, and therefore the slope measured is not a direct measure of the fractal dimension. It is nevertheless still a key metric in describing the mesoscale structure in these systems. Table 2 gives the slope of a log-log plot for each of the simulated and experimental systems, where that slope is linear (simulations: $0.19<Q<0.35 \AA^{-1}$, experiment $0.1<Q<0.25 \AA^{-1}$ ).

Table 2: Measured slopes of log-log plot of experimental and simulated SANS scattering curves in the Porod region. 


\begin{tabular}{l|llcc}
\multicolumn{1}{l}{ TEMPERATURE } & ASPHC & ASPHLE & MIXED ASPHL & EXPERIMENT \\
\hline $20 / 27^{\circ} \mathrm{C}$ & 2.82 & 2.86 & 3.10 & 2.72 \\
$180^{\circ} \mathrm{C}$ & 2.09 & 2.21 & 2.20 & 2.28
\end{tabular}

\begin{abstract}
All the simulations show a clear decrease in the slope with increasing temperature, and very similar values as seen in the experimental scattering curves data. The $Q$-range over which the scattering curve reaches a plateau is related to the size of the scattering object. Although at these concentrations structure factor effects will play a role, and distances are best thought of as a correlation length rather than an aggregate size. ${ }^{47}$ The data clearly show that the correlation lengths in the simulation are smaller (on
\end{abstract} average) than in the experimental sample. As with the solid sample, there are two root causes to this discrepancy: Firstly, the limited system size that can be accessed through molecular simulation and secondly the lack of polydispersity in the molecular structures used. We note that the use of only a single molecular structure also gives rise to the shoulder in the simulated scattering data for asphaltene $C$ in the region $0.5<Q<1 \AA^{-1}$ due to correlations related to the fixed size of the molecule, which would not occur in a 
polydisperse system. For the intermediate $Q$-range data (lower half of Figure 7 ) the scattering is dominated by that from the 1-methylnaphthalene solvent. The change in peak position and width with temperature is relatively well reproduced by the simulation, but scattering intensities are not. This observation is being investigated further by an in depth study of the liquid structure of 1-methylnaphthalene using neutron scattering with isotopic substitution. 

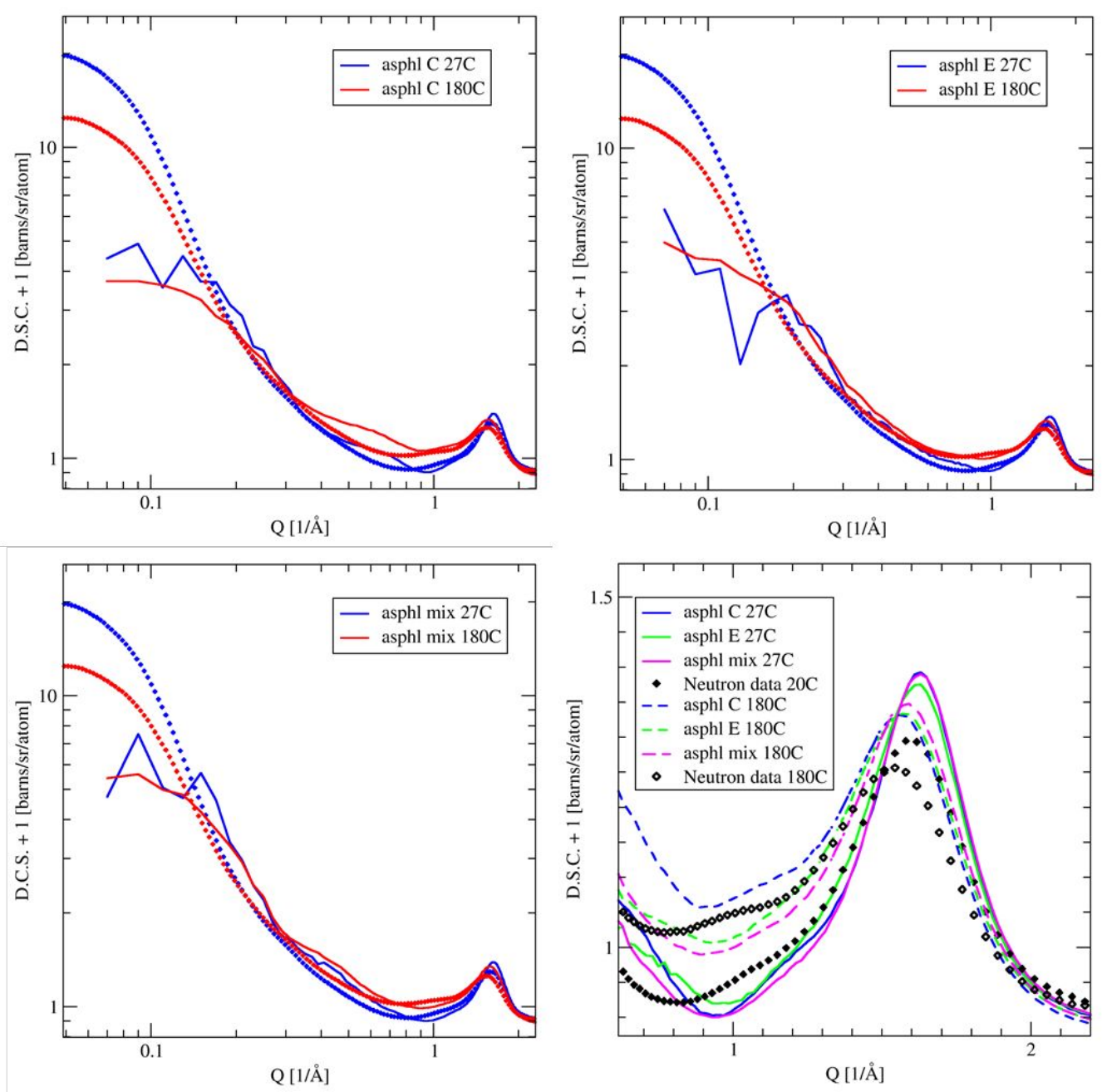

Figure 7: Total neutron differential scattering cross section (D.C.S.) for $25 w t \%$

Athabasca asphaltenes in 1-methylnaphthalene-d10 with comparison to calculated scattering from simulations of asphaltene $\mathrm{C}$ (top left), asphaltene $\mathrm{E}$ (top right) a mixture of all three asphaltenes (bottom left). Lower right plot shows the intermediate Q-range scattering in more detail and on a linear scale. 


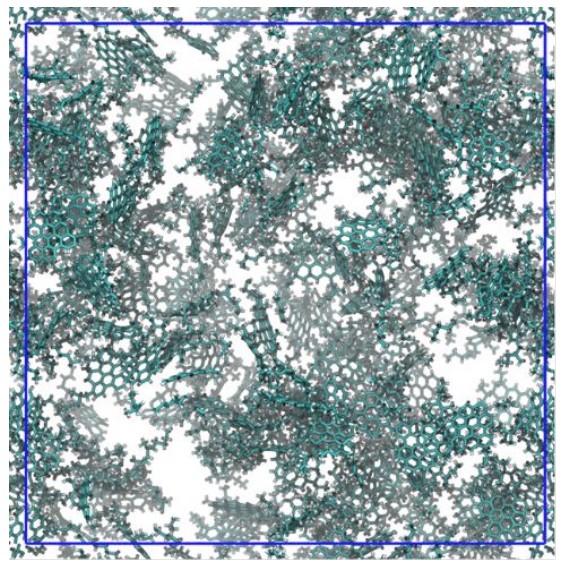

Small continental asphaltene (C)

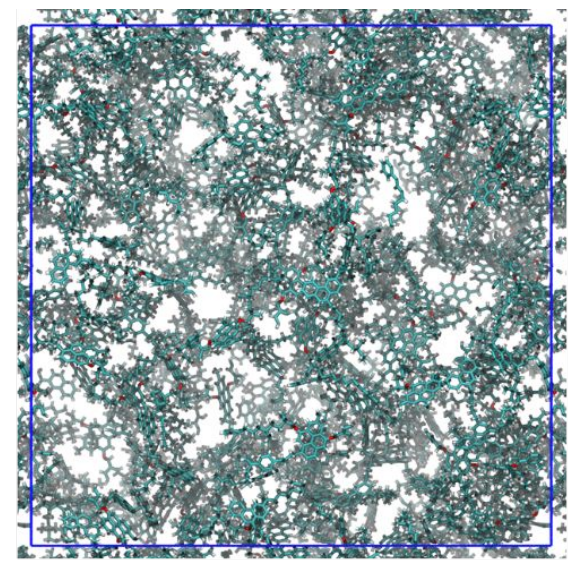

Archipelago asphaltene (E)

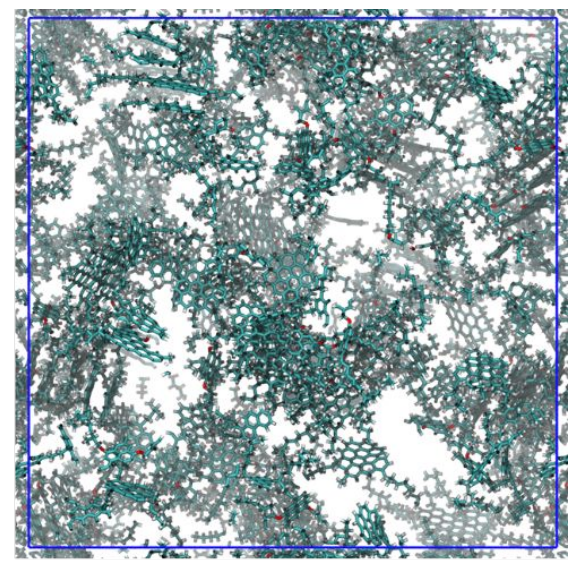

Mixed asphaltenes (C,D and E)

Figure 8: Snapshots of MD simulations of models asphaltenes in 1-methylnaphthalened10 at $300 \mathrm{~K}(27 \mathrm{C})$. For clarity solvent molecules are not shown.

A study of the simulation snapshots (Figure 8) hint at differences in aggregation between the molecules, with clear regions of visible aggregation for the asphaltene $C$ and mixed systems. Following the analysis used for solid asphaltenes we calculated the radial distribution functions for asphaltene aromatic carbon in the four simulations run, the results are shown in Figure 9. For the small continental asphaltene $C$ there is a clear short-range aromatic-aromatic interaction, with a peak in the $g(r)$ near $0.49 \mathrm{~nm}$, with no such peak visible for aromatic carbons in the archipelago asphaltene $\mathrm{E}$, and a very 
large peak seen for the mixed systems due to the presence of the large continental asphaltene $D$ in that system. It is notable that the peak in the $g(r)$ for asphaltene $C$ in solution (Figure 9) is greater than in the solid state (Figure 6), presumably due to packing constraints, where there is a need to maximize the number molecular contacts with a larger number of asphaltene molecules in the solid system than in solution. This observation could be grounds for future investigation of the effect of solvent on asphaltene aggregation properties, and that the solid-phase studies may not be representative of the structure in the dispersed phase. 


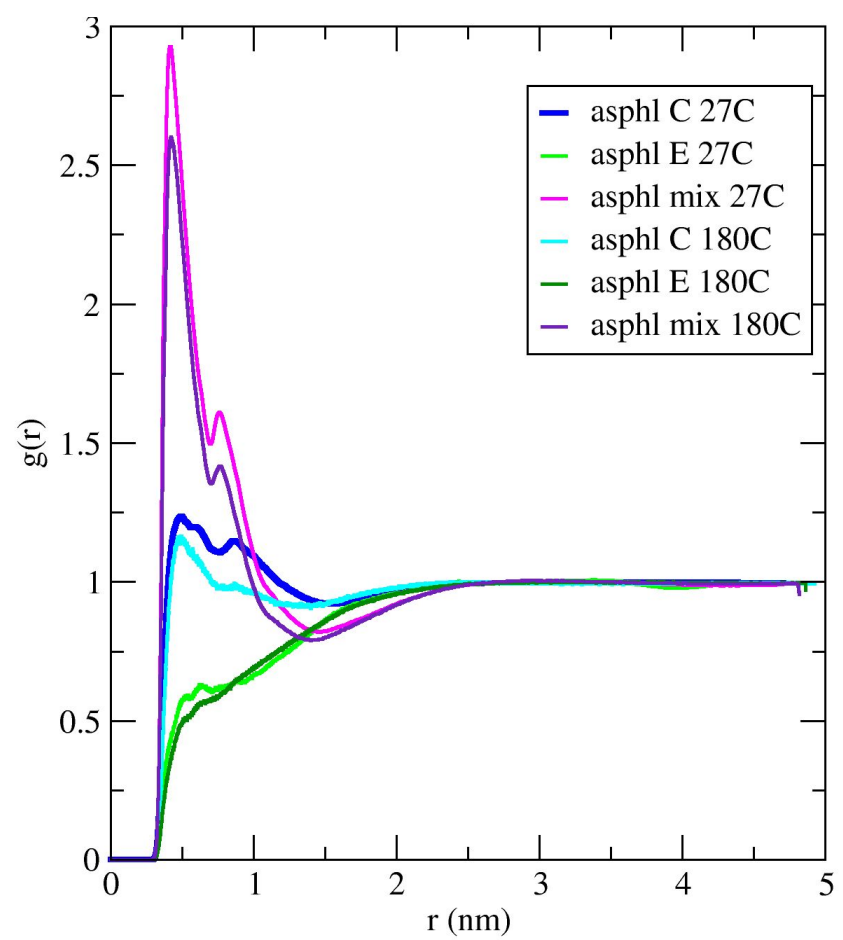

Figure 9: Radial distribution functions, $g(r)$, for aromatic carbons for simulations of asphaltenes $C, E$ and a mixture of $C, D$ and $E$ in 1-methylnaphthalene.

\section{Conclusions and Outlook}

This report details the first-ever comparison between atomistic molecular dynamics simulations and experimental total scattering measurements. From this comparison, it is 
clear that there is still room for improvement in the prediction of asphaltene aggregate structure from molecular dynamics simulations as presented here. As asphaltenes are immensely heterogeneous and complex, we do not anticipate exact matches, and there are some positive observations with respect to simulation approaches. The match to the data was reasonable in a few areas: In both the simulation of solid and solution dispersed asphaltenes the general form of the small-angle scattering curve was correct. It is particularly encouraging that for the dissolved asphaltenes the slope of the curves, and their modification with temperature matches the data well. This indicates that, at the length scales simulated, the fractal diffuse structure of the aggregation is well replicated.

Ultimately, the important outcomes of this preliminary study are to find where standard simulation methods do not reproduce the experimentally determined structure. The comparison of the solid simulations to the data point towards the fact that there is increased phase separation of the aromatic rich and poor molecules in the experimental system compared to the simulation. Therefore clearly the size of the system, and the timescales simulated are both limiting factors. The simulation boxes used in this study 
are very large, in the region $9-10 \mathrm{~nm}$ in length and containing around 80,000 atoms. For reference, a typical 400ns simulation took $\sim 2$ weeks on 96 compute cores (over 4 nodes). This approach is pushing the limits of what can currently be achieved by atomistic simulations in reasonable timescales. Coarse-graining methods ${ }^{52,53}$ will be key in allowing access to longer length scales in reasonable timescales, although how to compare the results to scattering data over a wide $Q$-range remains an open question. Secondly, as has been noted elsewhere ${ }^{29}$, polydispersity in the asphaltene molecular structures is vital to correctly replicating and predicting aggregation structure and should be included in any simulation looking to correctly predict asphaltene structure and phase behavior.

\section{AUTHOR INFORMATION}

\section{Corresponding Author}

*Email: tom.headen@stfc.ac.uk 


\section{ACKNOWLEDGEMENT}

The authors wish to thank the UK Engineering and Physical Sciences Research Council

(grant EP/R013195/1) and the US National Science Foundation (grant CBET-1821762)

for financial support. Neutron beamtime was provided by the UK Science and

Technology Facilities Council (STFC) through award number RB1710282 and molecular dynamics simulations were completed by the use of the STFC SCARF computing resource.

\section{REFERENCES}

(1) Headen, T. F.; Boek, E. S. E. S.; Jackson, G.; Totton, T. S. T. S.; Müller, E. A. A. Energy \& Fuels 2017, 31 (2), 1108.

(2) Santos, D.; Souza, W.; Santana, C.; Lourenço, E.; Santos, A.; Nele, M. ACS Omega 2018, 3(4), 3851.

(3) Yang, F.; Tchoukov, P.; Dettman, H.; Teklebrhan, R. B.; Liu, L.; Dabros, T.; Czarnecki, J.; Masliyah, J.; Xu, Z. Energy \& Fuels 2015, 29 (8), 4783. 
(4) Jian, C.; Liu, Q.; Zeng, H.; Tang, T. Energy \& Fuels 2018, 32 (3), 3225.

(5) Ervik, Å.; Lysgaard, M. O.; Herdes, C.; Jiménez-Serratos, G.; Müller, E. A.; Munkejord, S. T.; Müller, B. J. Comput. Phys. 2016, 327, 576.

(6) Mohammed, S.; Mansoori, G. A. Energy \& Fuels 2018, 32 (4), 5409.

(7) Headen, T. F.; Boek, E. S. E. S. Energy \& Fuels 2011, 25 (2), 503.

(8) Xiong, Y.; Cao, T.; Chen, Q.; Li, Z.; Yang, Y.; Xu, S.; Yuan, S.; Sjöblom, J.; Xu, Z. J. Phys. Chem. C2017, $121(9), 5020$.

(9) Headen, T. F.; Boek, E. S. E. S. Energy \& Fuels 2011, 25 (2), 503.

(10) Zhu, X.; Wu, G.; Coulon, F.; Wu, L.; Chen, D. Energy \& Fuels 2018, acs. energyfuels.8b00470.

(11) Ungerer, P.; Rigby, D.; Leblanc, B.; Yiannourakou, M. Mol. Simul. 2014, 40 (1-3), 115.

(12) Goual, L.; Sedghi, M.; Wang, X.; Zhu, Z. Langmuir 2014. 
(13) Goual, L.; Sedghi, M. J. Colloid Interface Sci. 2015, 440, 23.

(14) Jorgensen, W. L.; Severance, D. L. J. Am. Chem. Soc. 1990, $112(12), 4768$.

(15) MacKerell, A. D.; Bashford, D.; Bellott, M.; Dunbrack, R. L.; Evanseck, J. D.;

Field, M. J.; Fischer, S.; Gao, J.; Guo, H.; Ha, S.; Joseph-McCarthy, D.; Kuchnir,

L.; Kuczera, K.; Lau, F. T. K.; Mattos, C.; Michnick, S.; Ngo, T.; Nguyen, D. T.;

Prodhom, B.; Reiher, W. E.; Roux, B.; Schlenkrich, M.; Smith, J. C.; Stote, R.;

Straub, J.; Watanabe, M.; Wiórkiewicz-Kuczera, J.; Yin, D.; Karplus, M. J. Phys.

Chem. B 1998, $102(18), 3586$.

(16) Wang, J.; Wolf, R. M.; Caldwell, J. W.; Kollman, P. A.; Case, D. A. J. Comput.

Chem. 2004, 25 (9), 1157.

(17) Jorgensen, W. L.; Maxwell, D. S.; TiradoRives, J. J. Am. Chem. Soc. 1996, 118 (45), 11225.

(18) Headen, T. F.; Cullen, P. L.; Patel, R.; Taylor, A.; Skipper, N. T. Phys. Chem. Chem. Phys. 2018, 20 (4), 2704. 
(19) Keen, D. A.; IUCr. J. Appl. Crystallogr. 2001, 34 (2), 172.

(20) Soper, A. K. Mol. Simul. 2012, 38 (14-15), 1171.

(21) Headen, T. F.; Howard, C. A.; Skipper, N. T.; Wilkinson, M. A.; Bowron, D. T.;

Soper, A. K. J. Am. Chem. Soc. 2010, 132 (16), 5735.

(22) Soper, A. K. Phys. Rev. B 2005, $72(10), 104204$.

(23) Fu, C.-F.; Tian, S. X. J. Chem. Theory Comput. 2011, 7(7), 2240.

(24) Klein, G. C.; Angström, A.; Rodgers, R. P.; Marshall, A. G. Energy \& Fuels 2006, $20(2), 668$.

(25) Mullins, O. C.; Sabbah, H.; Eyssautier, J.; Pomerantz, A. E.; Barré, L.; Andrews, A. B.; Ruiz-Morales, Y.; Mostowfi, F.; McFarlane, R.; Goual, L.; Lepkowicz, R.; Cooper, T.; Orbulescu, J.; Leblanc, R. M.; Edwards, J.; Zare, R. N. Energy \& Fue/s 2012, 26 (7), 3986.

(26) Headen, T. F.; Boek, E. S.; Jackson, G.; Totton, T. S.; Müller, E. A. 2016. 
(27) Headen, T. F.; Boek, E. S. E. S.; Skipper, N. T. N. T. Energy and Fue/s 2009, 23 (3), 1220.

(28) Marshall, A. G.; Rodgers, R. P. Proc. Natl. Acad. Sci. U. S. A. 2008, 105 (47), 18090.

(29) Javanbakht, G.; Sedghi, M.; Welch, W. R. W.; Goual, L.; Hoepfner, M. P. J. Mol. Liq. 2018, 256, 382.

(30) Bowron, D. T.; Soper, A. K.; Jones, K.; Ansell, S.; Birch, S.; Norris, J.; Perrott, L.; Riedel, D.; Rhodes, N. J.; Wakefield, S. R.; Botti, A.; Ricci, M.-A.; Grazzi, F.; Zoppi, M. Rev. Sci. Instrum. 2010, 81 (3).

(31) Hoepfner, M. P.; Vilas Bôas Fávero, C.; Haji-Akbari, N.; Fogler, H. S. Langmuir 2013, $29(28), 8799$.

(32) Headen, T. F.; Boek, E. S. E. S.; Stellbrink, J.; Scheven, U. M. M. Langmuir 2009, $25(1), 422$.

(33) Eyssautier, J.; Levitz, P.; Espinat, D.; Jestin, J.; Gummel, J.; Grillo, I.; Barré, L. J. 
Phys. Chem. B 2011, $115(21), 6827$.

(34) Gawrys, K. L.; Kilpatrick, P. K. J. Colloid Interface Sci. 2005, 288 (2), 325.

(35) Soper, A. K. GudrunN and GudrunX: Programs for Correcting Raw Neutron and X-ray Diffraction Data to Differential Scattering Cross Section http://purl.org/net/epubs/work/56240 (accessed Aug 23, 2018).

(36) Soper, A. K. Mol. Phys. 2009, $107(16), 1667$.

(37) Soper, A. K. ISRN Phys. Chem. 2013, 2013, 1.

(38) Jorgensen, W. L.; McDonald, N. A. Theochem-Journal Mol. Struct. 1998, 424 (12), 145.

(39) Nose, S. Mol. Phys. 1984, 52, 255.

(40) Hoover, W. G. Phys. Rev. A 1985, 31, 1695.

(41) Parinnello, M.; Rahman, A. J. Appl. Phys. 1981, 52, 7182.

(42) Nose, S.; Klein, M. L. Mol. Phys. 1983, 50, 1055. 
(43) Abraham, M. J.; Murtola, T.; Schulz, R.; Páll, S.; Smith, J. C.; Hess, B.; Lindahl, E. SoftwareX 2015, 1-2, 19.

(44) Hess, B. J. Chem. Theory Comput. 2008, 4 (1), 116.

(45) Darden, T.; York, D.; Pedersen, L. J. Chem. Phys. 1993, 98 (12), 10089.

(46) Youngs, T. G. A. DLPUtils https://www.projectaten.com/dlputils.

(47) Sirota, E. B. Pet. Sci. Technol. 1998, 16(3-4), 415.

(48) Yen, T. F.; Erdman, J. G.; Pollack, S. S. Anal. Chem. 1961, 33 (11), 1587.

(49) Andersen, S. I.; Jensen, J. O.; Speight, J. G. Energy \& Fue/s 2005, 19 (6), 2371.

(50) Chacón-Patiño, M. L.; Rowland, S. M.; Rodgers, R. P. Energy \& Fuels 2017, 31 (12), 13509.

(51) Teixeira, J. J. Appl. Crystallogr. 1988, 21 (6), 781.

(52) Herdes, C.; Totton, T. S.; Müller, E. A. Fluid Phase Equilib. 2015, 406, 91.

(53) Jover, J. F.; Müller, E. A.; Haslam, A. J.; Galindo, A.; Jackson, G.; Toulhoat, H.; 
Nieto-Draghi, C. Energy \& Fuels 2015, 29 (2), 150121165549008. 